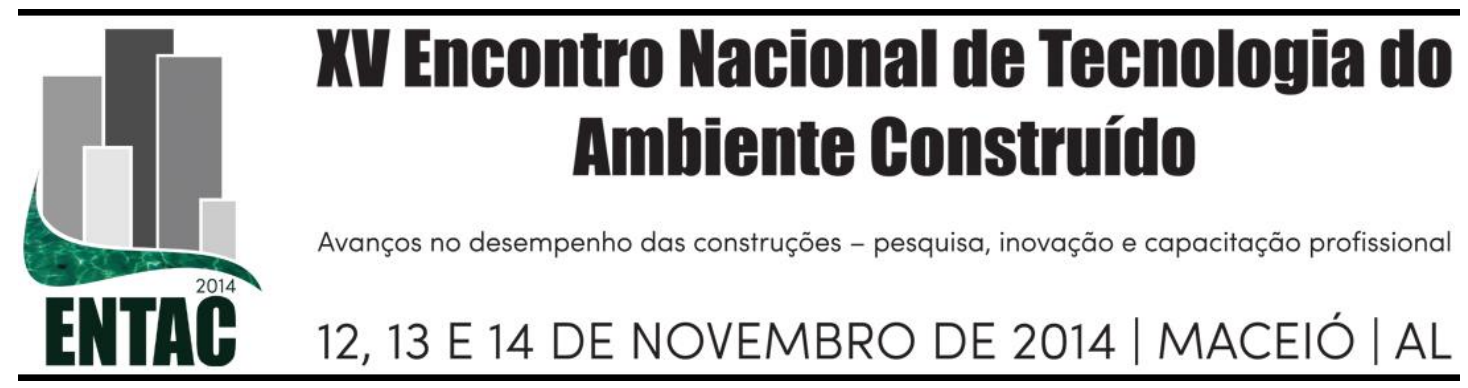

\title{
ANÁLISE DO POTENCIAL DE ECONOMIA DE ENERGIA ELÉTRICA NOS PAVIMENTOS DE GARAGEM DE UMA EDIFICAÇÃO ETIQUETADA, CONSIDERANDO O PRÉ- REQUISITO DE CONTRIBUIÇÃO DA LUZ NATURAL DO RTQ-C.
}

\author{
BRANDÃO, Guilherme Caixeta (1); SOUZA, Roberta Viera Gonçalves de (2 \\ (1)Universidade Federal de Minas Gerais, (031) 3409-8823, guicbrandao@gmail.com (2) Universidade \\ Federal de Minas Gerais, robertavgs2@gmail.com
}

\begin{abstract}
RESUMO
Os métodos de avaliação fornecidos pelo RTQ-C para a determinação da eficiência do sistema de iluminação artificial determinam que avaliação de classificação seja feita pela densidade de potência instalada por atividade ou uso da edificação $\left(\mathrm{W} / \mathrm{m}^{2}\right)$, o que permite a determinação de limites para carga instalada de acordo com a atividade para cada classificação que varia de A e E. São exigidos prérequisitos específicos como a Divisão de Circuitos, Desligamento Automático e Contribuição da Luz Natural. O pré-requisito de contribuição de luz natural implica em mudanças na concepção dos projetos luminotécnico, elétrico e de automação das edificações. Assim, este trabalho, parte de uma dissertação de mestrado em andamento, tem como objetivo analisar o potencial de redução de consumo de energia elétrica em pavimentos de garagem de edificações comerciais, a partir da aplicação do pré-requisito de Contribuição de Luz Natural. O método deste trabalho baseia-se no estudo de caso de uma edificação com área etiquetada para iluminação de $19.680 \mathrm{~m}^{2}$, e que atende a todos os pré-requisitos de avaliação de eficiência do sistema de iluminação do RTQ-C. Os resultados mostraram uma redução potencial de $8,85 \%$ do consumo total de energia elétrica das garagens, valor considerável se analisarmos o consumo mensal de $14.777 \mathrm{kWh} /$ mês apenas nestes ambientes. Desta forma, observou-se que a separação do acionamento das fileiras de luminárias próximas às aberturas, em conjunto com rotinas de automação possibilitam a redução considerada significativa do consumo de energia elétrica em edificações comerciais.
\end{abstract}

Palavras-chave: Iluminação natural, Iluminação artificial, Eficiência Energética, Redução de consumo

\begin{abstract}
The evaluation methods provided by RTQ - $C$ to determine the artificial lighting system efficiency determines that review of classification by density of installed power per activity or use of the building $\left(W / m^{2}\right)$, which allows the determination of limits for installed load according to activity for each rating ranging from A to E. Specific prerequisites are required as the Division of Circuits, Auto Power Off and Natural Light Contribution. The prerequisite natural light contribution implies changes in the light design, electrical and automation of buildings projects. This work, part of a dissertation in progress, aims to analyze the potential for reducing electricity consumption in garage floors of commercial buildings, from the application of prerequisite of Natural Light Contribution. The method of this work is based on the study case of a labeled building with $19.680 \mathrm{~m}^{2}$ for lighting area, and meets all prerequisites for
\end{abstract}


evaluating the lighting system efficiency of the RTQ-C. The results showed a potential reduction of $8.85 \%$ of the total electricity consumption of the garages, considerable value if analyze the monthly consumption of 14,777 kWh/month only in these areas. Thus, it was observed that the separation of the drive next to the openings fixtures rows, in combination with automation schedules allow significantly the reduction of the energy consumption in commercial buildings.

Keywords: Daylighting, Artificial Lighting, Energy Efficiency, Potential Energy Savings

\section{INTRODUÇÃ̃O}

A crise do setor energético brasileiro ocorrido em 2001 com o apagão deixou em alerta as autoridades do setor. O Governo Federal iniciou uma série de investimentos com o objetivo de desenvolver pesquisas que apontassem diretrizes para a racionalização de energia no país, com a promulgação da Lei $\mathrm{n}^{\circ}$. 10.295, que dispõe sobre a Política Nacional de Conservação e Uso Racional de Energia (BRASIL, 2001a) e o Decreto $n^{\circ}$ 4059 de 19 de dezembro de 2001 (BRASIL, 2001b) que regulamentou a Lei estabelecendo "níveis máximos de consumo de energia, ou mínimos de eficiência energética, de máquinas e aparelhos consumidores de energia fabricados ou comercializados no País, bem como as edificações construídas".

A análise global da distribuição dos consumos energéticos revela que grande parte da energia gerada no Brasil é utilizada pelos edifícios comerciais, residenciais e de serviços, consumindo 49,8\% da oferta de energia elétrica do país (BEN, 2012). A iluminação é responsável por, aproximadamente, $24 \%$ do consumo de energia elétrica no setor residencial, $44 \%$ no setor comercial e serviços públicos e $1 \%$ no setor industrial.

Segundo Vianna e Gonçalves (2001 apud Fonseca et al, 2012) projetos desenvolvidos considerando a busca da luz natural chegam a alcançar a iluminância requisitada nos interiores de $80 \%$ a $90 \%$ das horas diurnas do ano economizando consideráveis quantidades de energia elétrica.

A iluminação natural em si não resulta em economia direta de energia, sua economia se dá através da redução do requerimento de iluminação artificial através da integração entre os sistemas de luz natural e artificial e através de sistemas de controle da iluminação natural. (FERREIRA e SOUZA, 2009)

O Regulamento Técnico da Qualidade do Nível de Eficiência Energética de Edifícios Comerciais, de Serviços e Públicos (RTQ-C), publicado pela Portaria INMETRO n. ${ }^{\circ}$ 372, de 17 de setembro de 2010, complementada em 2012 pela portaria INMETRO n. ${ }^{\circ}$ 17, de 16 de janeiro de 2012, estabelece parâmetros para a avaliação da eficiência da envoltória, do sistema de iluminação e do sistema de condicionamento de ar das edificações. A iluminação da edificação é avaliada através dos requisitos e prérequisitos específicos do sistema de iluminação. $\mathrm{O}$ método de avaliação aplicado pelo RTQ-C foi baseado na norma americana ASHRAE/IESNA Standard 90.1 - Energy Standard for Buildings Except Low-Rise Residential Buildings - e define, para a 
realidade brasileira, as densidades de potência limite para cada tipo de edifício ou ambiente em função da classificação almejada.

Segundo Ramos e Lamberts (2010), "este novo método determina a densidade de potência máxima instalada por atividade ou uso da edificação $\left(\mathrm{W} / \mathrm{m}^{2}\right)$, o que permite a determinação de limites para carga instalada de acordo com a atividade. Além disso, reduz a possibilidade de superdimensionamento do sistema e conduz a uma avaliação mais rápida por parte do Laboratório de Inspeção (sic)". O método anterior de avaliação do desempenho energético de sistemas de iluminação do RTQ-C consistia em determinar a densidade de potência de iluminação relativa final (DPIRF) de determinado projeto luminotécnico, para cada índice de ambiente $(\mathrm{K})$, comparando-a a limites pré-estabelecidos de densidades de potência de iluminação relativa limite (DPIRL) para cada nível pretendido (RAMOS e LAMBERTS, 2010).

Além dos limites de potência instalada estabelecidos pelo regulamento, são exigidos pré-requisitos específicos como a Divisão de Circuitos, Desligamento Automático e Contribuição da Luz Natural. O ítem 4.1.2 do RTQ-C determina:
4.1.2. Contribuição da luz natural
Ambientes com abertura(s) voltada(s) para o ambiente externo ou para átrio não coberto ou de cobertura translúcida e que contenham mais de uma fileira de luminárias paralelas à(s) abertura(s) devem possuir um controle instalado, manual ou automático, para o acionamento independente da fileira de luminárias mais próxima à abertura, de forma a propiciar o aproveitamento da luz natural disponível. Unidades de edifícios de meios de hospedagem são exceção a este pré-requisito. (Instituto Nacional de Metrologia, Normalização e Qualidade Industrial (INMETRO). Portaria $\mathrm{n}^{\mathrm{o}}$ 372, de 17 de setembro de 2010. Requisitos Técnicos da Qualidade para o Nível de Eficiência Energética de Edifícios Comerciais, de Serviços e Públicos (RTQ-C). Brasília, DF, 2010c. p. 38.)

Estes critérios, apesar de objetivarem a redução do consumo de energia elétrica através do controle dos sistemas de iluminação, tem se mostrado um complicador no processo de etiquetagem das edificações. O pré-requisito de contribuição de luz natural implica em mudanças na concepção dos projetos luminotécnicos, elétrico e de automação das edificações e por isto, seu potencial de redução de consumo de energia elétrica deve ser investigado de forma a avaliarmos sua pertinência.

\section{REVISÃO BIBLIOGRÁFICA}

As edificações, que compreendem, basicamente, os setores residencial, comercial, institucional e público responderam com aproximadamente $197 \mathrm{TWh}$, ou seja, 44\% do consumo de energia elétrica do Brasil. Grande parte dessa energia é destinada a prover conforto ambiental aos usuários, por meio de sistemas artificiais de iluminação, climatização e aquecimento de água. O potencial técnico de economia em edificações 
existentes é estimado em $25 \%$, enquanto que em prédios novos pode alcançar até $50 \%$, ou seja, quando se considera a eficiência energética nas edificações desde a concepção do projeto. (ELETROBRAS, 2013)

Ferreira e Souza (2009) desenvolveram uma revisão da literatura sobre o potencial de economia de energia em iluminação em edifícios comerciais. A Tabela 1 mostra que a variação do potencial de economia de energia através do uso da iluminação natural está relacionado ao tipo do sistema de controle utilizado e que os potenciais variam de $18 \%$ a $68 \%$ quando usados sensores on-off e de $2 \%$ a $77 \%$ quando usados sistemas de dimerização.

Tabela 1 - Resumo dos dados coletados sobre o potencial de economia de energia da iluminação natural

\begin{tabular}{|c|c|c|c|c|c|c|c|c|c|}
\hline \multirow{3}{*}{ Autor } & \multirow{3}{*}{ Ano } & \multirow{3}{*}{ Localidade } & \multirow{3}{*}{ Latitude } & \multirow{3}{*}{$\begin{array}{l}\text { Tipologia da } \\
\text { edificação }\end{array}$} & \multirow{3}{*}{ Sistema de controle da luz natural } & \multicolumn{4}{|c|}{ Potencial de economia(\%) } \\
\hline & & & & & & \multicolumn{2}{|c|}{ Fachada Sul } & \multicolumn{2}{|c|}{ Fachada Norte } \\
\hline & & & & & & Min. & Máx. & Min. & Máx. \\
\hline \multirow{2}{*}{ Brekke e Hansen } & \multirow{2}{*}{1995} & \multirow{2}{*}{ - } & \multirow{2}{*}{ - } & \multirow{2}{*}{ - } & Dimmer & 30 & 40 & 30 & 40 \\
\hline & & & & & Dimmer & 20 & 30 & 20 & 30 \\
\hline Szerman & 1993 & Alemanha & . & Escritórios & Dimmer & - & 77 & - & 77 \\
\hline Alves et al. & 1998 & Belo Horizonte (Brasil) & $19^{\circ} 55^{\prime} \mathrm{S}$ & Comercial & Dimmer & 12,7 & 64,3 & 12,7 & 64,3 \\
\hline Li et al. & 2006 & Hong Kong & $22^{\circ} 15^{\prime} \mathrm{N}$ & Escolas & Dimmer & \multicolumn{4}{|c|}{33} \\
\hline Lee e Selkowitz & 2006 & Nova York (Estados Unidos) & $40^{\circ} 47^{\prime} \mathrm{N}$ & Escritórios & Dimmer & \multicolumn{4}{|c|}{$>60$} \\
\hline Li e lam & 2002 & Hong Kong & $22^{\circ} 15^{\prime} \mathrm{N}$ & Escolas & Dimmer & \multicolumn{4}{|c|}{ Em torno de 70} \\
\hline \multirow{2}{*}{ Atife Galasiu } & \multirow{2}{*}{2002} & Quebec & $46^{\circ} 48^{\prime} \mathrm{N}$ & \multirow{2}{*}{ Escritórios } & Dimmer & \multicolumn{4}{|c|}{ Acima de 63,5} \\
\hline & & Ottawa & $45^{\circ} 24^{\prime} \mathrm{N}$ & & Controle automático on/off & \multicolumn{4}{|c|}{68} \\
\hline Ghisi e lamberts & 1997 & Florianópolis (Brasil) & $27^{\circ} 35^{\prime} \mathrm{S}$ & Escolas & Controle on/off & 18 & 50 & 18 & 50 \\
\hline Galasivet al. & 2003 & Ontario (Canadá) & $43^{\circ} 50^{\prime} \mathrm{N}$ & Escritórios & Dimmer e controle automático on/off & 50 & 60 & 50 & 60 \\
\hline Li etal. & 2004 & Hong Kong (China) & $14^{\circ} 6^{\prime} \mathrm{N}$ & Escritórios & Dimmer e controle automático on/off & - & 33 & - & 33 \\
\hline \multirow{3}{*}{ Roisin et al. } & \multirow{3}{*}{2007} & Atenas (Grécia) & $37^{\circ} \mathrm{g}^{\prime} \mathrm{N}$ & \multirow{3}{*}{ Escritórios } & pimmer com controle individual de luminária e sensor de presenç̧ & 61 & 63 & 57 & 59 \\
\hline & & Bruxelas (Bélgica) & $50^{\circ} \mathrm{g}^{\prime} \mathrm{N}$ & & pimmer com controle individual de luminária e sensor de presenç̧ & 54 & 56 & 51 & 53 \\
\hline & & Estocolmo (Suécia) & $59^{\circ} 65^{\prime} \mathrm{N}$ & & pimmer com controle individual de luminária e sensor de presenç̧ & 51 & 53 & 47 & 49 \\
\hline Opdal e Brekke & 1995 & Trondheim (Noruega) & $63^{\circ} 36^{\prime} \mathrm{N}$ & Escritórios & immer com controle individual de luminária e sensor de presenç̧ & 26 & 35 & 2 & 29 \\
\hline Knight & 1999 & Reino Unido & Entre $40^{\circ} \mathrm{e} 61^{\circ} \mathrm{N}$ & . & Dimmer com controle individual de luminária & 44 & 76 & 44 & 76 \\
\hline Embrechts e Van Bellegem & 1997 & Bélgica, Paises Baixos e Luxemburgo & Entre $40^{\circ} \mathrm{Ne} 51^{\circ} 5^{\prime} \mathrm{N}$ & Escritórios & Dimmer com controle individual de luminária & 20 & 40 & 20 & 40 \\
\hline Onaygil e Güller & 2003 & Istambul (Turquia) & $40 \circ 58^{\prime} \mathrm{N}$ & Escritórios & Dimmer com controle individual de luminária & 21 & 45 & 21 & 45 \\
\hline
\end{tabular}

Fonte: FERREIRA e SOUZA, 2009

Dos trabalhos citados acima, vale destacar o artigo desenvolvido por Roisin et al (2008) em que eles comparam o potencial de economia de energia gasta em iluminação em escritórios utilizando diferentes sistemas de controle (de presença e dimmer), para 3 localidades da Europa (Bruxelas, Estocolmo e Atenas). Observou-se que o uso de sistemas de controle apresenta uma alta economia de energia, dependendo fortemente da orientação e da localização do ambiente.

No cenário nacional, Ghisi e Lamberts (1997) realizaram um levantamento das condições de iluminação natural em salas de aula do Centro Tecnológico da Universidade Federal de Santa Catarina, objetivando avaliar o potencial de economia de energia elétrica através da redução do uso de iluminação artificial. As medições foram realizadas em 4 salas de aula de condições e dimensões equivalentes. O sistema das salas de aula, composto por 4 luminárias perpendiculares ao plano das janelas, 
permitiam o desligamento das duas fileiras de luminárias próximas às janelas ou apenas da fileira mais próxima às janelas, de acordo com a disponibilidade de luz natural. Através da aplicação deste tipo de controle on/off obteve-se uma economia de eletricidade em iluminação de $50 \%$ no período de primavera e verão. Já no período de inverno e outono a economia foi de $18 \%$.

Já o trabalho desenvolvido por Alves et al. (1998) diz respeito à integração dos sistemas de iluminação artificial e natural, aplicada ao estudo de caso do Mercado Central de Belo Horizonte. A integração dos sistemas neste caso se deu através de dimerizadores conjugados com os sensores de iluminação. No estudo realizado, o controlador aciona a alimentação do circuito para os níveis programados de $33 \%, 50 \%$ ou plena carga. Com esse sistema, em termos de consumo de energia mensal, alcançou-se uma economia de $64,3 \%$ no primeiro pavimento e de $12,7 \%$ no segundo pavimento da edificação.

A integração entre os sistemas de iluminação artificial e natural dependem, no entanto, da adequação do projeto arquitetônico e dos sistemas de abertura, bem como da disponibilidade de luz natural em função das condições climáticas. Souza (2008) afirma que um bom projeto de iluminação natural irá englobar aspectos relacionados à adequação de dimensionamento e forma das aberturas para melhor aproveitamento da luz, e irá fazer uso de sistemas de iluminação artificial complementares, apenas quando necessário, para obter níveis adequados de iluminação para o desenvolvimento das tarefas visuais requeridas no ambiente.

\section{OBJETIVO}

Este trabalho tem como objetivo analisar o potencial de redução de consumo de energia elétrica em edificações comerciais, a partir da aplicação do pré-requisito de Contribuição de Luz Natural, referente à avaliação do sistema de iluminação do RTQ-C usando como estudo de caso um ambiente de garagem.

\section{MÉTODO}

O método deste trabalho baseia-se no estudo de caso de uma edificação, etiquetada na etapa de projeto, que atende a todos os pré-requisitos de avaliação de eficiência do sistema de iluminação do RTQ-C. Foi calculado consumo mensal de energia elétrica, feita a aplicação dos pré-requisitos e avaliado o acionamento da iluminação artificial através de determinação de rotina de ocupação, estabelecida a partir da divisão de circuitos presente nos ambientes de garagem da edificação.

\section{RESULTADOS E DISCUSSÃO}

O objeto de análise deste artigo, uma edificação comercial etiquetada na fase de projeto, teve seus projetos luminotécnico, elétrico e de automação avaliados pela equipe de consultoria do GRA e desta forma, pode-se acompanhar as modificações sofridas pelo 
projeto no processo para melhor adequação aos requisitos de eficiência energética do RTQ-C.

A edificação, de aproximadamente $33.000 \mathrm{~m}^{2}$, possui 19 andares tipo, além de um pavimento térreo com centro de convenções e espaço de academia, e quatro níveis de garagem. Como os pavimentos tipos e as áreas de centro de convenções e academia serão entregues aos proprietários para acabamento posterior, apenas as áreas de uso comum da edificação foram etiquetadas. Com $19.000 \mathrm{~m}^{2}$ as áreas de uso comum possuem um sistema de iluminação com $65.000 \mathrm{~W}$ de potência instalada.

Os $34.500 \mathrm{~W}$ de potência instalada nos $15.000 \mathrm{~m}^{2}$, distribuídos em quatro níveis de garagem, e que representam $53 \%$ da potência instalada nas áreas etiquetadas do edifício, serão objeto de análise em relação à pertinência do atendimento ao pré-requisito de Contribuição da Luz Natural, levando em conta às dimensões dos ambientes, a tecnologia utilizada, a necessidade de mudança de conceito de projeto e operação, bem como o potencial de redução de consumo proveniente de tais alterações.

O primeiro projeto luminotécnico elaborado para a edificação previa uma malha paralela de luminárias distribuída por todo o pavimento, com a utilização de conjuntos compostos por lâmpadas fluorescentes tubulares T5 de $28 \mathrm{~W}$ e reatores eletrônicos dimerizáveis com interface DALI, um dos mais eficientes do mercado.

O projeto elétrico, por sua vez, foi desenvolvido de forma a setorizar o acionamento do sistema de iluminação, tornando mais lógica a instalação e a operação do sistema. Os circuitos foram dispostos alternadamente, com um circuito vigia instalado entre dois "circuitos regulares". O sistema de automação da edificação é o responsável pela operação dos circuitos regulares, enquanto o circuito vigia permanece acionado $24 \mathrm{~h}$, por segurança.

Com o processo de etiquetagem da edificação, a análise de potência instalada nas garagens apontava densidade de potência instalada dentro dos limites estabelecidos pelo RTQ-C para classificação $\mathrm{A}$, no entanto, como o projeto elétrico não previa $\mathrm{o}$ acionamento separado das fileiras de luminárias próximas às aberturas, para atendimento ao pré-requisito de Contribuição da Luz Natural, todo este setor foi classificado como C.

Foram necessárias alterações no projeto elétrico de forma a criar uma nova lógica de divisão dos circuitos que possibilitasse o acionamento das fileiras de luminárias próximas às aberturas, independente das demais luminárias do ambiente. Das 180 luminárias previstas para o $5^{\circ}$ Subsolo, 35 foram instaladas no novo circuito, assim como no $4^{\circ}$ Subsolo. O $3^{\circ}$ Subsolo possui 266 luminárias, sendo 43 separadas no novo circuito, enquanto o $2^{\circ}$ Subsolo, maior pavimento de garagem da edificação, das 400 luminárias previstas em projeto, 42 foram separadas para atendimento ao pré-requisito.

A separação das luminárias em circuitos específicos para o atendimento do pré-requisito de iluminação natural do RTQ-C, além de impactar na criação de novos circuitos, 
acarretou em alterações no sistema de automação da edificação, com a inserção de uma nova rotina de acionamento para estas luminárias.

Das 1026 luminárias instaladas nos quatro pavimentos de garagem, 155 tiveram seu acionamento separado das demais luminárias dos pavimentos, gerando nove novos circuitos na edificação. Dos $31.806 \mathrm{~W}$ de potência instalada nas garagens, $4.805 \mathrm{~W}$ representam as luminárias que atendem ao pré-requisito, representando $15,1 \%$ do total.

Tabela 2 - Quantidade de luminárias com acionamento separado

\begin{tabular}{|c|c|c|c|c|}
\hline Pavimento & $\begin{array}{c}\text { Quantidade de } \\
\text { luminárias }\end{array}$ & $\begin{array}{c}\text { Potência } \\
\text { total } \\
\text { instalada (w) }\end{array}$ & $\begin{array}{c}\text { Quantidade de } \\
\text { luminárias com } \\
\text { acionamento separado }\end{array}$ & $\begin{array}{c}\text { Potência } \\
\text { total } \\
\text { instalada (w) }\end{array}$ \\
\hline $5^{\mathbf{0}}$ Subsolo & 180 & 5580 & 35 & 1085 \\
\hline $4^{\mathbf{0}}$ Subsolo & 180 & 5580 & 35 & 1085 \\
\hline $3^{\mathbf{0}}$ Subsolo & 266 & 8246 & 43 & 1333 \\
\hline $2^{\mathbf{o}}$ Subsolo & 400 & 12400 & 42 & 1302 \\
\hline Total & $\mathbf{1 0 2 6}$ & $\mathbf{3 1 8 0 6}$ & $\mathbf{1 5 5}$ & $\mathbf{4 8 0 5}$ \\
\hline
\end{tabular}

Fonte: Elaborado pelo autor

Para análise do potencial de redução de consumo que o atendimento ao pré-requisito de Contribuição da Luz Natural trará à edificação, foi necessário estabelecer as rotinas de funcionamento dos circuitos instalados nas garagens. Primeiramente foram estabelecidas as rotinas da edificação com base no primeiro projeto elétrico desenvolvido para o edifício. Neste foram identificados duas rotinas distintas: circuitos "regulares" com funcionamento entre $6 \mathrm{~h}$ e $22 \mathrm{~h}$; e circuitos vigias com funcionamento 24 horas por dia.

Tabela 3 - Consumo mensal de energia do projeto original

\begin{tabular}{|c|c|c|c|c|}
\hline Circuito & $\begin{array}{c}\text { Quantidade de } \\
\text { luminárias }\end{array}$ & $\begin{array}{c}\text { Potência total } \\
\text { instalada (W) }\end{array}$ & $\begin{array}{c}\text { Horas de } \\
\text { funcionamento/mês }\end{array}$ & $\begin{array}{c}\text { Potência total } \\
\text { utilizada } \\
\text { (kWh/mês) }\end{array}$ \\
\hline Regular & 898 & 27838 & 480 & 13362,24 \\
\hline Vigia & 128 & 3968 & 720 & 2856,96 \\
\hline Total
\end{tabular}

Fonte: Elaborado pelo autor

Posteriormente foram estabelecidas as rotinas da edificação, considerando as alterações necessárias para o atendimento ao pré-requisito de Contribuição da Luz Natural. Foram identificadas 3 rotinas diferentes para os circuitos, sendo: circuitos "regulares" com funcionamento das $6 \mathrm{~h}$ às $22 \mathrm{~h}$; circuitos separados pelo pré-requisito que ficarão desligados entre $8 \mathrm{~h}$ e $17 \mathrm{~h}$ e funcionarão das $17 \mathrm{~h}$ às $22 \mathrm{~h}$; circuitos vigias com funcionamento 24 horas por dia. 
Tabela 4 - Consumo mensal de energia das garagens com Contribuição de Luz Natural

\begin{tabular}{|c|c|c|c|c|}
\hline Circuito & $\begin{array}{c}\text { Quantidade } \\
\text { de luminárias }\end{array}$ & $\begin{array}{c}\text { Potência total } \\
\text { instalada (W) }\end{array}$ & $\begin{array}{c}\text { Horas de } \\
\text { funcionamento/ mês }\end{array}$ & $\begin{array}{c}\text { Potência total } \\
\text { utilizada } \\
\text { (kWh/mês) }\end{array}$ \\
\hline Regular & 743 & 23033 & 480 & 11055,84 \\
\hline Pré-requisito & 155 & 4805 & 180 & 864,90 \\
\hline Vigia & 128 & 3968 & 720 & 2856,96 \\
\hline Total & \multicolumn{4}{|l}{} \\
\hline
\end{tabular}

Fonte: Elaborado pelo autor

Após a criação dos novos circuitos e das novas rotinas de automação do sistema de iluminação, observou-se uma redução de consumo mensal de $1153,2 \mathrm{kWh} / \mathrm{mês}$, representando uma economia de $8,85 \%$ do consumo total de energia elétrica das garagens.

Para avaliarmos o potencial de redução de consumo considerando todo o sistema de iluminação das áreas comuns da edificação, é necessário estabelecer uma rotina de operação para estas áreas. Desta forma, estabeleceu-se o funcionamento do sistema semelhante ao funcionamento dos circuitos regulares das garagens, de $6 \mathrm{~h}$ às $22 \mathrm{~h}$.

Tabela 5 - Consumo mensal de energia das áreas de uso comum

\begin{tabular}{|c|c|c|c|}
\hline Circuito & $\begin{array}{c}\text { Potência total } \\
\text { instalada }(\mathbf{W})\end{array}$ & $\begin{array}{c}\text { Horas de } \\
\text { funcionamento/mês }\end{array}$ & $\begin{array}{c}\text { Potência total } \\
\text { utilizada }(\mathbf{k W h} / \mathbf{m e ̂ s})\end{array}$ \\
\hline $\begin{array}{c}\text { Demais áreas de uso } \\
\text { comum }\end{array}$ & 33194 & 480 & 15933,12 \\
\hline
\end{tabular}

Fonte: Elaborado pelo autor

A partir das rotinas estabelecidas e do atendimento ao pré-requisito de Contribuição da Luz Natural a análise do potencial de consumo gerado na edificação como um todo, indica uma economia mensal no consumo de energia elétrica de aproximadamente $4,67 \%$.

Os resultados apresentados acima foram obtidos considerando apenas o controle do sistema de iluminação projetado para a edificação. Este sistema é baseado no controle por uma central de automação, com controle on/off, sem a participação ativa do usuário e/ou adaptabilidade às condições de uso ou ambientais.

\section{CONSIDERAÇÕES FINAIS}

A necessidade de implantar a eficiência energética nas edificações impactará diretamente na forma como os projetos arquitetônicos e complementares são concebidos. A introdução de novas tecnologias não poderá ser a única responsável pela diminuição do impacto do parque construído no meio ambiente. Os projetistas deverão absorver tais tecnologias e repensar em algumas das práticas projetuais utilizadas, da mesma forma que os usuários das edificações deverão se adaptar as novas rotinas de operação destas. 
Os sistemas de iluminação artificial estão diretamente relacionados com a qualidade e com a maneira com que o usuário percebe o espaço. Desta forma, o projeto de um sistema de iluminação eficiente deve levar em conta não somente o consumo energético, mas também as implicações deste na rotina do usuário.

A prática de consultoria do GRA mostra que o pré-requisito de Contribuição da Luz Natural tem gerado consideráveis mudanças na concepção dos projetos elétricos, que devem conciliar os projetos luminotécnicos aos requisitos do RTQ-C.

A coleta de dados realizada por Ferreira e Souza (2009) aponta para economia, em ambientes de escritório, que varia de $2 \%$ a $77 \%$ dependendo do tipo do sistema de controle utilizado e da orientação da edificação. Sendo que, para escritórios com sistemas de controle on/off, semelhante ao utilizado na edificação, os valores variam de $18 \%$ a $68 \%$. Tais percentuais de economia, no entanto, foram obtidos em ambientes de escritórios e, por isso, não devem ser diretamente comparados com os percentuais obtidos neste trabalho.

Os valores obtidos a partir da análise dos dados da edificação estudada demonstram considerável potencial de redução de consumo de energia elétrica apenas com a separação de uma fileira de luminárias próxima às aberturas no caso de garagens em edifícios comerciais que possuam aberturas em seu perímetro. Este potencial, no entanto, deverá ser comparado com outras situações semelhantes para verificação do desempenho do sistema.

A análise de outras 3 edificações etiquetadas com consultoria do GRA aponta que a potencia instalada nos sistemas de iluminação das garagens destes edifícios, representa de $13 \%$ a $31 \%$ da potência instalada nas áreas de uso comum destas edificações. Tais percentuais são significativos quando se analisa o consumo destas áreas e mostram que há um significativo potencial de redução de consumo de energia elétrica com a adoção do pré-requisito de Contribuição da Luz Natural para estas áreas.

Para tanto é necessário considerar, no desenvolvimento do projeto arquitetônico, um dimensionamento adequado dos sistemas de aberturas, bem como verificar a disponibilidade de luz natural em função das condições climáticas, uma vez que estes fatores influenciam diretamente no potencial de redução do consumo de energia elétrica através do aproveitamento da iluminação natural.

As possibilidades de aumentar a economia de energia nestas áreas poderiam ser testadas a partir da inserção de outros sistemas de controle do sistema de iluminação, uma vez que o sistema de automação com interface DALI não leva em consideração a disponibilidade de luz natural no ambiente.

A simulação computacional para verificação da oferta de luz natural nos ambientes poderia contribuir para o aumento da economia de energia elétrica, à medida que serviria de indicador para a viabilidade de separação de mais de uma fileira de luminárias próximas às aberturas, aumentando ainda mais o potencial de economia. 
Por fim, a pertinência da aplicação do pré-requisito de Contribuição da Luz Natural na edificação estudada, deverá ser referendada pela análise das rotinas de operação desta edificação quando em operação, para que se possa fazer uma comparação dos resultados obtidos.

\section{REFERÊNCIAS}

ALVES, T. P.; LARA, V. C. D.; VIANA JÚNIOR, G. F.; VONO, C. P.; ASSIS, E. S.; SOUZA, R. V. G. Análise de Desempenho e Otimização do Sistema de Iluminação Artificial do Mercado Central. In. Núcleo de Pesquisa em Tecnologia da Arquitetura e Urbanismo. BRASIL. 1998.

Ministério do Desenvolvimento, Indústria e Comércio Exterior. Instituto Nacional de Metrologia, Normalização e Qualidade Industrial (INMETRO). Portaria $\mathrm{n}^{0} 372$, de 17 de setembro de 2010. Requisitos Técnicos da Qualidade para o Nível de Eficiência Energética de Edifícios Comerciais, de Serviços e Públicos (RTQ-C). Brasília, DF, 2010c.

CARLO,J.,FERREIRA,R.,LAMBERTS,R. A Aplicação do Regulamento Técnico da Qualidade para sistemas de iluminação. In: X Encontro Nacional e VI Encontro Latino Americano de Conforto no Ambiente Construído. Natal, 2009.10p.

ELETROBRAS; PROCEL. Relatório de resultados do Procel 2013 - ano base 2012. Rio de Janeiro, 2013.

FERREIRA, C.C, SOUZA, R.V.G. Investigação sobre o potencial de economia de energia da iluminação natural. In. X Encontro Nacional e VI Encontro Latino Americano de Conforto no Ambiente Construído. Natal, 2009.4p.

FONSECA, R. W.; DIDONÉ, E. L.; PEREIRA, F. O. R. Modelos de predição da redução do consumo energético em edifícios que utilizam a iluminação natural através de regressão linear multivariada e redes neurais artificiais. Ambiente Construído, Porto Alegre, v. 12, n. 1, p. 163-175, jan./mar. 2012.

GHISI, E.; LAMBERTS, R. Avaliação das Condições de Iluminação Natural nas Salas de Aula da Universidade Federal de Santa Catarina. In.: I Encontro Nacional sobre Edificações e Comunidades Sustentáveis, Canela, 1997.

RAMOS,G.,LAMBERTS,R. Relatório Técnico do Método de avaliação do sistema de iluminação do RTQ-C. Relatório LabEEE UFSC/Neoenergia/UFMG/UNB, 2010.19p.

ROISIN, B.; BODART, M.; DENEYER, A.; D'HERDT, P. Lighting energy savings in offices using differente control systems and their real consumption. Energy and Buildings 40, 514$523,2008$.

SOUZA, R.V.G. Luz Natural no projeto arquitetônico. Revista Lume - $31^{\text {a }}$ Edição. Brasil. 2008 


\section{AGRADECIMENTOS}

Os autores gostariam de agradecer à FAPEMIG pelo apoio financeiro para participação no evento e à CAPES pelo apoio financeiro para o desenvolvimento da pesquisa. 